\title{
Efeito do processo de desidratação osmótica a pulso de vácuo na transferência de massa e nas propriedades reológicas e de cor de fatias de manga
}

Influence of pulsed vacuum osmotic dehydration on mass transfer, color and rheological properties of mango slices

\author{
Ana Paula ITO ${ }^{1}$, Marina CAVENAGHI ${ }^{1}$, Cinthya BERTOLDO² ${ }^{2}$ Kil Jin PARK ${ }^{2}$, Miriam Dupas HUBINGER ${ }^{1 *}$
}

\begin{abstract}
Resumo
O presente trabalho teve como objetivo estudar as propriedades reológicas, de cor e a cinética do processo de desidratação osmótica, a pulso de vácuo, de fatias de manga. As fatias de manga foram imersas em soluções hipertônicas de sacarose, nas concentrações de 45,55 e $65{ }^{\circ} \mathrm{Brix}$ com temperatura controlada $\left(20\right.$ e $\left.30^{\circ} \mathrm{C}\right)$. No início do processo, aplicou-se ou não um pulso de vácuo, utilizando as pressões de 50 e 100 mbar por 10 minutos. As frutas foram desidratadas por 5 horas, sendo avaliadas em diferentes tempos em relação à perda de peso, perda de água, ganho de sólidos, $\mathrm{a}_{\mathrm{w}}$, cor e propriedades reológicas (tensão e deformação na ruptura). O aumento da concentração e da temperatura da solução causou um aumento na perda de peso e de água enquanto que a incorporação de solutos foi favorecida em baixas concentrações e altas temperaturas e com a aplicação do pulso de vácuo. O amaciamento do produto, caracterizado pela diminuição da tensão máxima de ruptura, e o aumento da deformação na ruptura da amostra ocorreram nas condições de maior incorporação de solutos. Não houve alteração dos parâmetros de cor das mangas durante o processo de desidratação.

Palavras-chave: Tommy Atkins; tensão na ruptura; deformação na ruptura; conservação; cinética; croma.
\end{abstract}

\begin{abstract}
The aim of this work was to study rheological and colour properties and process kinetics of mango slices subject to pulsed vacuum osmotic dehydration. The slices were immersed in osmotic sucrose solutions $\left(45,55\right.$ and $\left.65{ }^{\circ} \mathrm{Brix}\right)$ at 20 or $30^{\circ} \mathrm{C}$. At the beginning, a sub-atmospheric pressure was applied or not to the solid-liquid system (50 or 100 mbar of pressure) for 10 minutes followed by an atmospheric pressure operation up to 5 hours. Fruit samples were evaluated after pre-determined times in terms of weight loss, water loss, solids gain, $\mathrm{a}_{\mathrm{w}}$, colour and rheological properties (stress and strain at rupture). The concentration and temperature increases lead to a weight and water reduction. Solid gain was favoured by lower concentration and a higher temperature and by the pulsed vacuum application. The sample softening, which is characterized by a reduction of maximum stress at rupture, and the increased stress on strain at rupture ocurred at the great solids updake conditions. The colour parameters did not change during the dehydration process.

Keywords: Tommy Atkins; stress at rupture; strain at rupture; conservation; kinetics; chroma.
\end{abstract}

\section{Introdução}

A manga é uma das frutas tropicais mais populares do mundo sendo nativa do sudeste da Ásia, onde se concentra a maior parte da produção mundial. O Brasil figura entre os 10 maiores produtores mundiais da fruta, ainda que o País responda por apenas um milhão de toneladas, da produção total mundial de 28 milhões de toneladas ${ }^{10}$. A manga é fonte de fibras e vitaminas, tais como a C e a pró-vitamina A (beta-caroteno). Entretanto, é pouco comercializada mundialmente, sendo a maior parte da produção consumida nos mercados internos. É uma fruta climatérica e perecível, que necessita de cuidados específicos para a sua conservação, trazendo dificuldades na pós-colheita nos países produtores. No mercado nacional, a manga é comercializada quase que exclusivamente na forma in natura, embora também possa ser encontrada nas formas de suco integral e polpa congelada.

O processo de desidratação osmótica, que consiste na imersão do produto em soluções concentradas de açúcar ou sal, promove a remoção parcial da água e a possível incorporação

\footnotetext{
Departamento de Engenharia de Alimentos,

Faculdade de Engenharia de Alimentos,

Universidade Estadual de Campinas - UNICAMP, CP 6121, CEP 13083-862,

Campinas - SP, Brasil

E-mail:mhub@fea.unicamp.br

Faculdade de Engenharia Agrícola, Universidade Estadual de Campinas,

Campinas - SP, Brasil

*A quem a correspondência deve ser enviada
}

de solutos. Pode ser utilizado com o objetivo de modificar a composição dos alimentos, permitindo a adição de ingredientes de interesse nutricional, sensorial ou de preservação. No processo de desidratação osmótica, são obtidas frutas de alta umidade, de boa qualidade nutricional, com a preservação do sabor e de outras propriedades sensoriais e com características similares ao produto fresco ${ }^{1}$.

O fenômeno de transferência de massa entre o produto e o meio desidratante é estritamente afetado pela natureza do produto (espécie, variedade, nível de maturação, porosidade, forma e tamanho, pré-tratamento) e pelas variáveis do processo (pré-tratamento, pressão, temperatura, natureza e concentração do agente osmótico, agitação, aditivos, tempo de processo) que exercem influência sobre a transferência de massa e sobre a qualidade do produto final ${ }^{3,15,21}$.

Tradicionalmente, o processo osmótico é conduzido à pressão atmosférica, entretanto diversos autores como FITO $^{13}$, ESCRICHE et al. ${ }^{9}$, PANADÉS et al..$^{22}$ entre outros, tem dado destaque à impregnação a vácuo de alimentos, como uma alternativa inovadora para os processos osmóticos difusivos. A desidratação osmótica a pulso de vácuo (PVOD - pulsed vacuum osmotic dehydration) consiste na aplicação de vácuo no sistema sólido-solução, por um curto período no início do processo, para retirar parte do ar presente no interior dos poros do alimento. Com a recuperação da pressão do sistema, o líquido que está em contato com o alimento penetra no interior 
dos poros, devido aos gradientes macroscópicos de pressão e à capilaridade $^{13}$. Este processo envolve uma rápida alteração na composição do alimento com conseqüências nas propriedades fisicas e de transporte do tecido do alimento.

Os trabalhos relacionados à técnica de PVOD indicam melhora no processo de transferência de massa, maior economia quando comparado ao uso de pressão atmosférica ou pressão contínua de vácuo, além de proporcionar tempos mais curtos

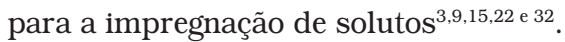

Na desidratação osmótica, a perda de água e de sólidos naturais do alimento e a incorporação de sólidos são responsáveis por modificações nas propriedades viscoelásticas do tecido, devido à plasticidade da estrutura da amostra após o tratamento. Entre as principais alterações ocorridas no processo, estão a perda de turgor da célula, alterações na lamela média e na resistência da parede celular, mudanças nas frações de volume de ar e líquido na amostra, além de variações no tamanho e forma do alimento $^{5}$. A aplicação de um pulso de vácuo no processo de desidratação osmótica pode provocar danos mecânicos na estrutura celular, como a separação celular, associada à deformação da amostra que ocorre quando o pulso de vácuo é aplicado.

Visando estudar o processo de desidratação osmótica a pulso de vácuo (PVOD) de fatias de manga, foram variadas a temperatura e a concentração da solução e a aplicação ou não de um pulso de vácuo no sistema. Analisou-se a perda de peso e de água, ganho de sólidos, $\mathrm{a}_{\mathrm{w}}$, propriedades reológicas (tensão e deformação na ruptura) e propriedades de cor (luminosidade e croma) durante o processo, com o objetivo de encontrar as melhores condições de trabalho.

\section{Material e métodos}

\subsection{Material}

As mangas da variedade Tommy Atkins foram adquiridas no CEASA/Campinas em caixas contendo de 11 a 13 frutos, com aproximadamente $6 \mathrm{~kg}$ no total. As frutas foram selecionadas de acordo com o grau de maturação (11-13 ${ }^{\circ}$ Brix), tamanho e formato $(552,4 \pm 54,1 \mathrm{~g}, 11,9 \pm 0,5 \mathrm{~cm}$ de comprimento e $10,0 \pm 0,4 \mathrm{~cm}$ diâmetro).

A matéria-prima foi caracterizada quanto à composição química centesimal segundo $\mathrm{AOAC}^{2}$ (Tabela 1). Os resultados obtidos para a composição química da manga foram próximos aos apresentados pela TACO (Tabela Brasileira de Composição de Alimentos $)^{28}$.

Tabela 1. Composição química da manga (Tommy Atkins var.).

\begin{tabular}{lcc}
\hline \multicolumn{1}{c}{ Análise (\%) } & $\begin{array}{c}\text { Valor médio } \pm \\
\text { desvio padrão }\end{array}$ & Valor médio (\%) ${ }^{28}$ \\
\hline Umidade em base úmida & $85,98 \pm 1,73$ & 85,8 \\
Açúcares totais & $11,42 \pm 1,63$ & 12,8 \\
Açúcares redutores & $3,86 \pm 0,12$ & - \\
Fibras & $1,19 \pm 0,17$ & 2,1 \\
Proteína (Nx6,25) & $0,68 \pm 0,09$ & 0,9 \\
Cinzas & $0,29 \pm 0,03$ & 0,3 \\
Lipídios & $0,45 \pm 0,07$ & 0,2 \\
\hline
\end{tabular}

As frutas foram sanitizadas através da lavagem em água corrente e depois foram mergulhadas por 10 minutos em uma solução contendo o Desinfetante Clorado para Verduras e Frutas Sumaveg (Diversey Lever, SP, Brasil, Componente químico: Dicloro S. Triazinatriona Sódica Dihidratada com $3 \%$ de cloro ativo ${ }^{23}$ ) na concentração de $0,66 \%\left(p \cdot v^{1}\right)$. A seguir, foram descascadas manualmente e as duas partes, paralelas à semente, foram cortadas. Estas partes foram fatiadas em $0,9 \mathrm{~cm}$ de espessura com o auxílio de uma guia de acrílico. A largura das fatias foi padronizada em $5 \mathrm{~cm}$ e a altura variou entre 2 e $3 \mathrm{~cm}$.

As soluções de sacarose utilizadas no processo osmótico foram preparadas com água destilada e sacarose comercial (Copersucar União, Piracicaba, SP, Brasil) variando-se as concentrações, de acordo com as condições estabelecidas em cada ensaio realizado (Tabela 2). Devido ao funcionamento do equipamento piloto, a proporção mínima de fruta:solução foi de 1:35 (p. $\left.\mathrm{p}^{-1}\right)$.

\subsection{Equipamento}

O processo de desidratação osmótica foi conduzido em um equipamento projetado para trabalhar à pressão atmosférica ou a vácuo ${ }^{32}$. O equipamento (Figura 1 ) é composto por uma câmara encamisada, de aço inox sanitário (A-240-304) com acesso pela parte superior. O sistema de termoregulação é constituído de um banho termoestático (Tecnal, model TE-184, Piracicaba, Brasil) que faz a circulação do fluido térmico (glicol) dentro da camisa do equipamento. Uma bomba centrífuga faz a recirculação da solução e a vazão é monitorada por um medidor tipo turbina. Para a formação de vácuo, uma bomba de vácuo acoplada ao sistema opera em diferentes valores de pressão reduzida (até $25 \mathrm{MPa}$ ou 250 mbar). O painel de controle foi programado para aquisição de dados da temperatura da solução osmótica, pressão de vácuo e vazão da solução durante o processo.

\subsection{Métodos}

\section{Procedimento experimental}

As fatias de manga foram pesadas e colocadas em bandejas de metal perfuradas e imersas na solução osmótica. Cada amostra foi feita em triplicata. Para o processo de desidratação osmótica a pulso de vácuo (PVOD), o sistema foi fechado e aplicou-se o vácuo pelo tempo e intensidade estabelecidos nos ensaios (Tabela 2). Após a aplicação do vácuo, o processo seguiu à pressão atmosférica. Em tempos pré-determinados $(0,25,0,5,1,2,3$ e 5 horas $)$, as amostras foram retiradas, drenadas, enxaguadas com água destilada e colocadas em papel absorvente, para remover o excesso de solução. Foram então pesadas e guardadas em potes herméticos para a realização das análises posteriores. Todas as análises foram realizadas em triplicada, exceto a análise das propriedades reológicas, realizada em quintuplicata. Para o processo de desidratação osmótica à pressão atmosférica, seguiu-se o mesmo procedimento, sem a aplicação de vácuo.

A cinética de desidratação foi estudada pelo acompanhamento da perda de peso (PP), perda de água (PA) e ganho de 


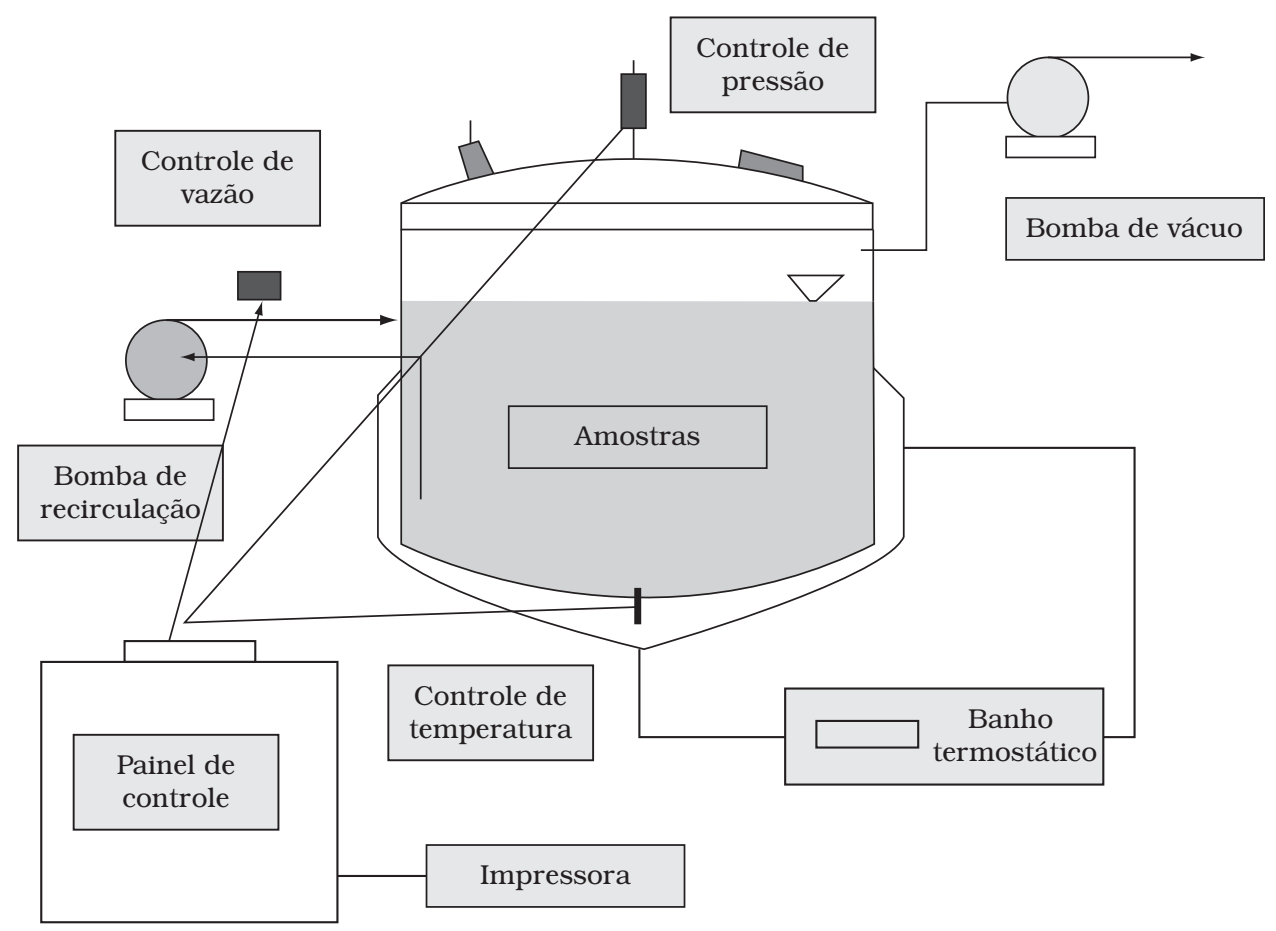

Figura 1. Esquema do equipamento de desidratação osmótica a pulso de vácuo.

sólidos (GS) e calculada pelas Equações de 1 a 3. As variações de peso foram obtidas, pesando as amostras antes e depois do tratamento osmótico e os conteúdos tanto de água quanto de sólidos foram obtidos utilizando os teores de umidade.

$P P(\%)=\frac{\left(M_{0}-M_{t}\right)}{M_{0}} 100$

$P A(\%)=\frac{\left(M a_{0}-M a_{t}\right)}{M_{0}} 100$

$G S(\%)=P A(\%)-P P(\%)$

sendo: $M_{0}=$ massa inicial do produto $(\mathrm{g})$;

$M_{t}=$ massa do produto a um tempo $\mathrm{t}(\mathrm{g})$;

$M a_{0}=$ conteúdo inicial de água no produto (g); e

$M a_{t}=$ conteúdo de água no produto a um tempo $\mathrm{t}(\mathrm{g})$.

Determinação de umidade, sólidos solúveis $e$ atividade de água

O teor de umidade foi determinado de acordo com o método $22.013 \mathrm{da} \mathrm{AOAC}^{2}$. Sólidos solúveis foram medidos com o refratômetro Zeiss West (Zeiss West, Berlim, Alemanha). A determinação da atividade de água foi realizada à temperatura de $25^{\circ} \mathrm{C}$, utilizando-se o equipamento Aqualab, modelo CX-2T (Decagon Devices Inc., Pullman, WA, EUA).

\section{Propriedades reológicas}

As propriedades reológicas estudadas foram a tensão e a deformação na ruptura, determinadas através de ensaio de compressão uniaxial a altas deformações da amostra, com auxílio do texturômetro Universal Testing Machine modelo TAXT2i (Texture Analyser, Stable Micro Systems, Surrey, Inglaterra). A compressão da amostra foi realizada utilizando uma placa cilíndrica de acrílico lubrificada de $60 \mathrm{~mm}$ de diâmetro, a uma velocidade de compressão de $1 \mathrm{~mm} / \mathrm{s}$ e $80 \%$ de deformação da amostra ${ }^{25}$. Os dados de força e altura fornecidos pelo equipamento foram convertidos à tensão $\left(\sigma_{\mathrm{H}}\right)$ e deformação $\left(\varepsilon_{\mathrm{H}}\right)$ de Hencky, de acordo com as Equações 4, 5 e 6 . A tensão e deformação na ruptura foram calculadas pelo valor do pico da curva tensão-deformação ${ }^{7}$.

$\sigma_{H}=\frac{F(t)}{A(t)}$

$\varepsilon_{H}=-\ln \left(\frac{H(t)}{H_{0}}\right)$

Sendo:

$$
\begin{aligned}
& \sigma_{H}=\text { tensão de Hencky (Pa); } \\
& \varepsilon_{H}=\text { deformação; e } \\
& F(t)=\text { força }(\mathrm{N}) \text { em função do tempo } \mathrm{t}(\mathrm{s}) .
\end{aligned}
$$

$A(t)=\frac{A_{0} H_{0}}{H(t)}$

Sendo: $A(t)=$ área $\left(\mathrm{m}^{2}\right)$ em função do tempo $(\mathrm{s})$;

$A_{0}=$ área inicial da amostra $\left(\mathrm{m}^{2}\right) ;$

$H_{0}=$ altura inicial da amostra (m); e

$H(t)=$ altura da amostra (m) em função do tempo (s). 
A tensão e a deformação na ruptura foram determinadas em quintuplicata ao longo do processo e para as amostras in natura. Para se desconsiderar a influência do grau de maturação da fruta, os resultados obtidos foram normalizados, ou seja, relacionados aos respectivos valores de tensão na ruptura para a fruta fresca utilizada em cada ensaio, por meio da razão entre eles.

\section{Avaliação de cor instrumental}

A cor foi avaliada através do sistema de leitura de três parâmetros, o CIELAB, proposto pela Commission Internationale de I'Eclairage (CIE) em 1971. Os parâmetros L*, a* e b* foram fornecidos pelo espectrofotômetro de bancada Color Quest II, marca Hunter Lab (Hunter Lab, Reston, EUA), em que L* define a luminosidade ( $\mathrm{L}^{*}=0$ preto e $\mathrm{L}^{*}=1$ branco) e $\mathrm{a}^{*} \mathrm{e} \mathrm{b}^{*}$ são responsáveis pela cromaticidade $\left(+\mathrm{a}^{*}\right.$ vermelho e $-\mathrm{a}^{*}$ verde ; $+b^{*}$ amarelo e -b* azul). Foi utilizado módulo de calibração de Reflectância Especular Incluída (RSIN) com o iluminante D65 e um ângulo de observação de $10^{\circ}$. Os parâmetros avaliados foram a luminosidade $\mathrm{L}^{*} \mathrm{e}$ o croma, que é calculado a partir da Equacão (7) e indica a saturação ou a intensidade da cor.

$C=\sqrt{\left(a^{*}\right)^{2}+\left(b^{*}\right)^{2}}$

\section{Resultados e discussão}

A Tabela 2 apresenta as condições estabelecidas para os ensaios realizados e os resultados obtidos para a perda de água (PA (\%)) e ganho de sólidos (GS (\%)) para os tempos mais significativos do processo (60, 120 e 300 minutos). Os parâmetros de processo utilizados foram: temperatura (20 e $\left.30{ }^{\circ} \mathrm{C}\right)$, concentração da solução $\left(45,55\right.$ e $\left.65^{\circ} \mathrm{Brix}\right)$ e a pressão de vácuo aplicada no sistema (p.atm. - sem aplicação de vácuo, 50 e 100 mbar de vácuo). A perda de peso (\%) apresentou valores semelhantes aos da perda de água, portanto esta variável não será apresentada neste trabalho.

\subsection{Variação da temperatura e da concentração}

O aumento da temperatura, de 20 para $30{ }^{\circ} \mathrm{C}$, causou o aumento tanto da perda de água quanto do ganho de sólidos, como pode ser observado na Figura 2. Essa grande influência na transferência de massa pode ser relacionada ao aumento da permeabilidade da membrana celular, causada pelas temperaturas mais elevadas ${ }^{17,31}$, e à redução da viscosidade da solução osmótica, que faz com que a resistência externa à transferência de massa seja menor, facilitando a transferência de solutos.

A transferência de massa, tanto de água quanto dos solutos, observada durante o contato entre o material e a solução osmótica é causada pela diferença de potencial químico, expressa pela atividade de água, entre o material e a solução ${ }^{27}$. O aumento da concentração de açúcares diminui a atividade de água da solução osmótica ( $\mathrm{a}_{\mathrm{w}}$ de 0,946 a $45{ }^{\circ} \mathrm{Brix}, 0,919$ a $55^{\circ} \mathrm{Brix}$ e 0,869 a $65{ }^{\circ} \mathrm{Brix}^{33}$ ), elevando a diferença de potencial entre o produto $\left(\mathrm{a}_{\mathrm{w}}\right.$ de 0,987 $\left.\pm 0,004\right)$ e a solução. Assim, o aumento da concentração da solução osmótica causou a maior perda de água do produto. Entretanto, concentrações mais elevadas evitaram o ganho de sólidos, como pode ser observado na Figura $2 b$, na qual se apresenta a cinética de ganho de sólidos nas concentrações de 45 e $65^{\circ}$ Brix.

O aumento da viscosidade da solução osmótica representou uma barreira para a transferência de massa da solução para a fruta ${ }^{16}$. Além disso, a intensa saída de água da fruta para a solução pode dificultar a impregnação de sólidos. SANJINEZARGANDOÑ ${ }^{26}$, TALENS et al. ${ }^{29}$ e FERRARI ${ }^{12}$ em seus trabalhos com goiabas, kiwis e melões, respectivamente, atribuíram o efeito negativo do aumento da concentração no ganho de sólidos à formação de uma densa camada de soluto na superfície do material, que atua como uma barreira à entrada de solutos.

\subsection{Aplicação do vácuo}

Para a concentração de sacarose de $45{ }^{\circ} \mathrm{Brix}$ a $20^{\circ} \mathrm{C}$, verificou-se que a aplicação de vácuo no início do processo não alterou a cinética de perda de água. Porém, para o ganho de sólidos, observou-se que a aplicação de 100 mbar de vácuo causou um aumento da incorporação de sólidos em comparação com o tratamento à pressão atmosférica, evidenciado a partir dos 120 minutos de processo (Figura 2b). A aplicação de 50 mbar não foi suficiente para causar alterações na cinética do processo.

Tabela 2. Condições utilizadas na desidratação osmótica das fatias de manga e resultados obtidos para perda de água (PA) e ganho de sólidos (GS) ao longo do processo.

\begin{tabular}{|c|c|c|c|c|c|c|c|c|c|}
\hline \multirow[t]{2}{*}{ Teste } & \multirow{2}{*}{$\begin{array}{c}\text { Temperatura } \\
\left({ }^{\circ} \mathrm{C}\right)\end{array}$} & \multirow{2}{*}{$\begin{array}{c}\text { Concentração } \\
\left({ }^{\circ} \text { Brix }\right)\end{array}$} & \multirow{2}{*}{$\begin{array}{c}\text { Pressão vácuo } \\
\text { (mbar) }\end{array}$} & \multicolumn{2}{|c|}{60 minutos } & \multicolumn{2}{|c|}{120 minutos } & \multicolumn{2}{|c|}{300 minutos } \\
\hline & & & & $\mathrm{PA}(\%)$ & GS(\%) & $\mathrm{PA}(\%)$ & GS(\%) & $\mathrm{PA}(\%)$ & GS(\%) \\
\hline 1 & 20 & 45 & p.atm. & 12,15 & 2,84 & 17,33 & 3,28 & 31,77 & 4,12 \\
\hline 2 & 20 & 45 & 50 & 10,40 & 2,02 & 18,17 & 2,45 & 30,67 & 4,87 \\
\hline 3 & 20 & 45 & 100 & 8,52 & 2,55 & 14,94 & 4,58 & 30,03 & 6,30 \\
\hline 4 & 20 & 55 & p.atm. & 15,05 & 2,50 & 20,42 & 2,49 & 33,53 & 4,66 \\
\hline 5 & 20 & 55 & 50 & 13,13 & 1,97 & 23,11 & 2,62 & 37,40 & 4,42 \\
\hline 6 & 20 & 55 & 100 & 16,01 & 3,50 & 23,71 & 3,83 & 37,47 & 4,21 \\
\hline 7 & 20 & 65 & p.atm. & 17,43 & 2,08 & 22,81 & 2,26 & 38,58 & 3,22 \\
\hline 8 & 20 & 65 & 50 & 19,95 & 2,57 & 23,26 & 3,36 & 37,51 & 3,74 \\
\hline 9 & 20 & 65 & 100 & 13,54 & 2,31 & 19,59 & 2,51 & 34,55 & 3,12 \\
\hline 10 & 30 & 45 & 100 & 18,24 & 3,96 & 26,20 & 5,27 & 39,95 & 6,33 \\
\hline 11 & 30 & 55 & p.atm. & 17,86 & 3,18 & 25,17 & 3,97 & 41,58 & 5,06 \\
\hline 12 & 30 & 55 & 100 & 20,39 & 3,48 & 29,77 & 4,20 & 44,33 & 5,09 \\
\hline 13 & 30 & 65 & 100 & 35,53 & 3,41 & 35,53 & 3,41 & 51,44 & 3,90 \\
\hline
\end{tabular}



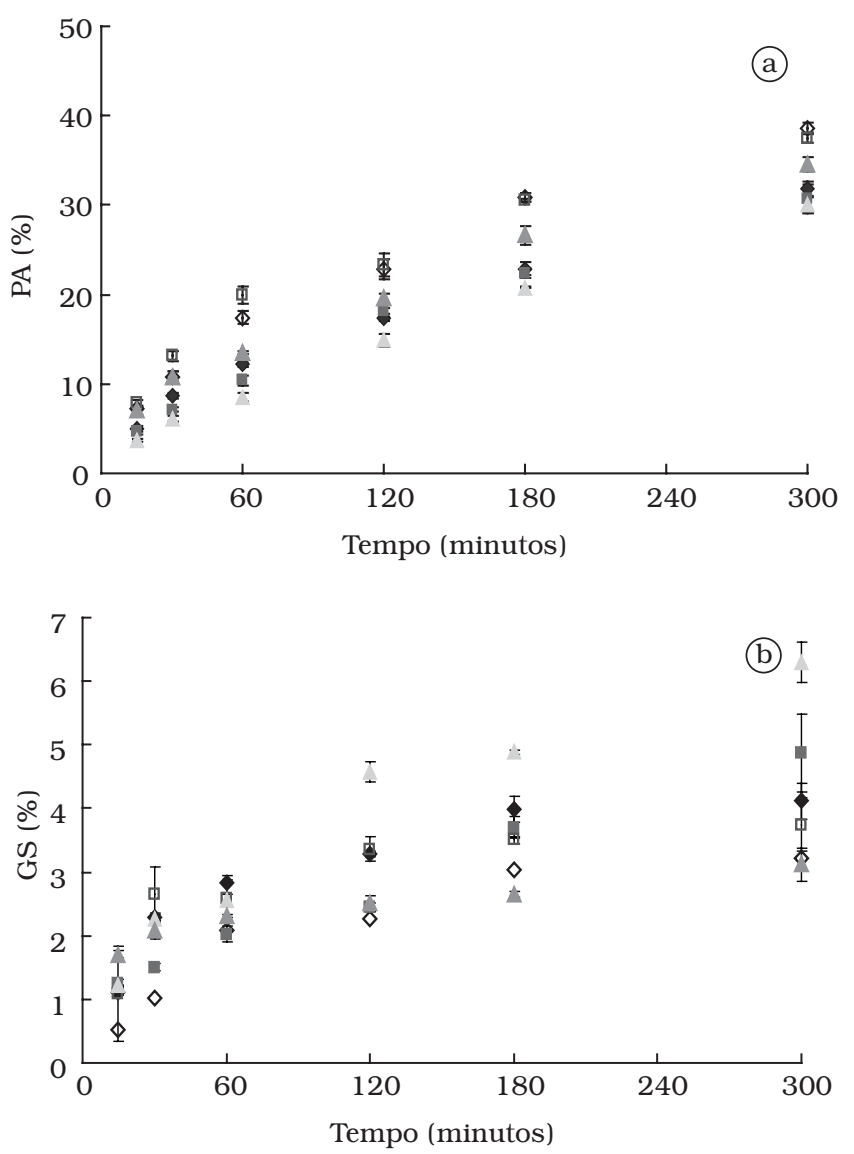

\begin{tabular}{|c|c|}
\hline $\begin{array}{l}45^{\circ} \text { Brix, p.atm } \\
45^{\circ} \mathrm{Brix}, 50 \mathrm{mbar} \\
45^{\circ} \mathrm{Brix}, 100 \mathrm{mbar}\end{array}$ & $\begin{array}{l}\diamond 65^{\circ} \mathrm{Brix}, \text { p.atm } \\
\text { 口 } 65^{\circ} \mathrm{Brix}, 50 \mathrm{mbar} \\
\triangle 65{ }^{\circ} \mathrm{Brix}, 100 \mathrm{mbar}\end{array}$ \\
\hline
\end{tabular}

Figura 2. a) Perda de água (PA (\%)); e b) Ganho de sólidos (GS(\%)) para 45 e $65^{\circ}$ Brix variando-se a pressão de vácuo aplicada.

Na concentração de $55^{\circ}$ Brix a $20^{\circ} \mathrm{C}$ (Figuras 3a e 3b), até o tempo de uma hora de processo, as condições estudadas apresentaram comportamento similar entre si. A partir deste tempo, observou-se que os tratamentos com aplicação de vácuo apresentaram maior perda de água em relação ao tratamento à pressão atmosférica. Para a temperatura de $30^{\circ} \mathrm{C}$, a aplicação de vácuo também causou maior perda de água do que o processo à pressão atmosférica. Entretanto, o ganho de sólidos foi semelhante ao tratamento a $20^{\circ} \mathrm{C}$.

No tratamento com solução a $65{ }^{\circ}$ Brix e $20^{\circ} \mathrm{C}$, o comportamento das condições sem aplicação de vácuo e com pulso de 50 mbar foi similar. A condição com aplicação de 100 mbar apresentou uma menor perda de água, possivelmente causada pela diferença entre os lotes adquiridos de matéria-prima. A aplicação de vácuo causou uma maior incorporação inicial de sólidos, que pode ser observada na Figura $2 \mathrm{~b}$ até os 60 minutos de processo. A partir desse tempo, a quantidade incorporada é semelhante, independentemente do tratamento aplicado.
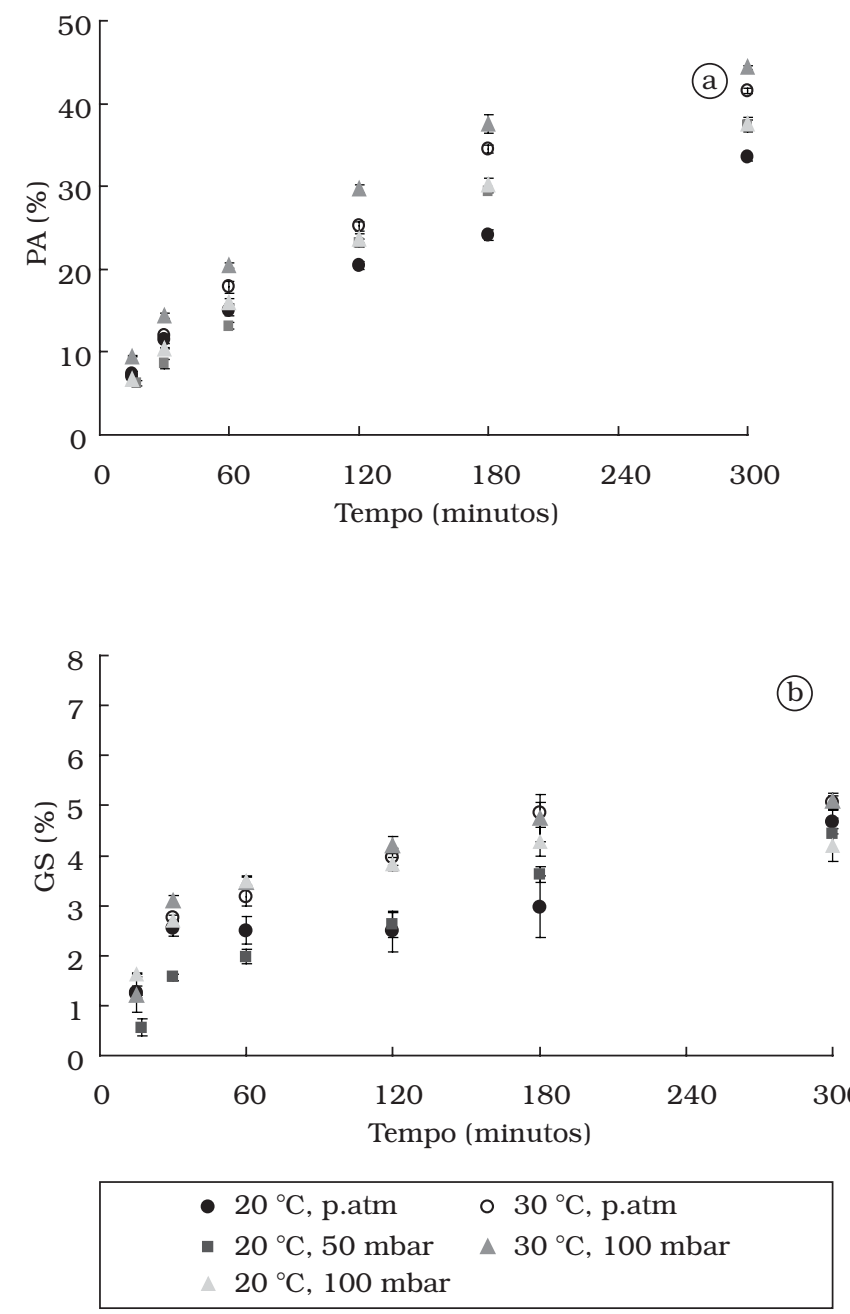

Figura 3. a) Perda de água (PA (\%)); e b) Ganho de sólidos (GS(\%))para $55^{\circ}$ Brix variando-se a pressão de vácuo aplicada e a temperatura do processo.

De maneira geral, o pulso de vácuo de 50 mbar não foi suficiente para causar alterações significativas nos processos estudados em comparação com o processo à pressão atmosférica. A aplicação de pulso de vácuo utilizando solução de $45^{\circ}$ Brix causou maior ganho de sólidos sem alterar a perda de água. A $55^{\circ} \mathrm{Brix}$, a aplicação de vácuo melhorou a perda de água e com 100 mbar de vácuo houve uma maior incorporação de solutos. A $65^{\circ} \mathrm{Brix}$, o pulso de vácuo provocou uma maior incorporação de sólidos enquanto que a perda de água não foi afetada. Para GIRALDO et al. ${ }^{15}$, as soluções de 45 e $65{ }^{\circ}$ Brix promoveram um maior ganho de sólidos nas mangas (Kent var.) submetidas à desidratação osmótica com aplicação de pulso de vácuo (50 mbar e 10 minutos) do que a solução de $55^{\circ}$ Brix.

ESCRICHE et al. ${ }^{8}$ variaram o tempo de aplicacação do pulso de vácuo (50 mbar) entre 5 e 15 minutos na desidratação osmótica de kiwi e não observaram diferença significativa na transferência de massa entre esses tratamentos. Somente houve 
diferença em comparação com o tratamento à pressão atmosférica. MÚJICA-PAZ et al. ${ }^{20}$ estudaram o volume impregnado de solução isotônica de sacarose em diversas frutas e relataram que o tempo de impregnação de vácuo (entre 3 e 45 minutos de vácuo seguido de 25 minutos à pressão atmosférica) teve um efeito linear no volume impregnado pela manga com a solução, porém a intensidade do vácuo (entre 135 e 674 mbar) não foi significativa. Em relação a outras frutas, o mesmo trabalho mostrou que o tempo e a intensidade de aplicação do vácuo foram variáveis significativas na impregnação de solução para o pêssego e mamão e, para a maçã, banana e melão, somente a intensidade do vácuo foi significativa.

Assim, a influência do tempo ou da intensidade do pulso de vácuo na impregnação da solução depende principalmente da estrutura do material e suas propriedades mecânicas. Outros autores relataram que o ganho de sólidos é maior quando se aplica um pulso de vácuo, em comparação com o processo à pressão atmosférica na desidratação osmótica em frutas, tais como a goiaba ${ }^{22}$, maçã $^{3}$, kiwi $^{8}$, manga ${ }^{15}$ entre outros. De acordo com FITO et al. ${ }^{14}$ e MÚJICA-PAZ et al. ${ }^{20}$, não é recomendável aplicar níveis elevados de pressão de vácuo, assim como longos tempos, pois isso causaria uma deformação irreversível no tecido da fruta, levando a uma redução da porosidade e diminuindo o volume livre disponível para a impregnação.

\subsection{Atividade de água}

A Tabela 3 apresenta os valores da atividade de água durante o processo. $\mathrm{A} \mathrm{a}_{\mathrm{w}}$ da fruta in natura variou entre $0,978 \mathrm{e}$ 0,990. A depressão da $\mathrm{a}_{\mathrm{w}}$ pode ser associada principalmente à perda de água e ao ganho de sólidos durante a desidratação osmótica. Sendo assim, o aumento da temperatura e da concentração favoreceu o abaixamento da $\mathrm{a}_{\mathrm{w}}$, enquanto que a aplicação do vácuo não provocou alterações. Observou-se um decréscimo nos valores de atividade de água da manga em todos os tratamentos, principalmente a partir dos 120 minutos de processo.

Apesar do processo de desidratação osmótica, por si só, não causar um abaixamento da $\mathrm{a}_{\mathrm{w}}$ suficiente para proteger $\mathrm{o}$ alimento contra a proliferação de microorganismos, o proces-

Tabela 3. Atividade de água ao longo do processo.

\begin{tabular}{cccccc}
\hline \multirow{2}{*}{ Teste } & \multicolumn{5}{c}{$\mathrm{a}_{\mathrm{w}}$} \\
\cline { 2 - 6 } & "In natura" & $60^{*}$ & $120^{*}$ & $180^{*}$ & $300^{*}$ \\
\hline 1 & 0,988 & 0,984 & 0,982 & 0,982 & 0,977 \\
2 & 0,983 & 0,983 & 0,981 & 0,980 & 0,975 \\
3 & 0,990 & 0,986 & 0,981 & 0,981 & 0,978 \\
4 & 0,987 & 0,982 & 0,980 & 0,980 & 0,978 \\
5 & 0,984 & 0,980 & 0,977 & 0,973 & 0,970 \\
6 & 0,990 & 0,987 & 0,985 & 0,984 & 0,977 \\
7 & 0,978 & 0,976 & 0,972 & 0,970 & 0,964 \\
8 & 0,990 & 0,977 & 0,976 & 0,972 & 0,972 \\
9 & 0,989 & 0,983 & 0,984 & 0,980 & 0,978 \\
10 & 0,989 & 0,981 & 0,979 & 0,977 & 0,973 \\
11 & 0,989 & 0,986 & 0,983 & 0,981 & 0,973 \\
12 & 0,989 & 0,984 & 0,981 & 0,974 & 0,973 \\
13 & 0,985 & 0,975 & 0,973 & 0,970 & 0,969 \\
\hline${ }^{* m i n u t o s}$ & & & & &
\end{tabular}

Ciênc. Tecnol. Aliment., Campinas, 27(supl.): 54-63, ago. 2007 


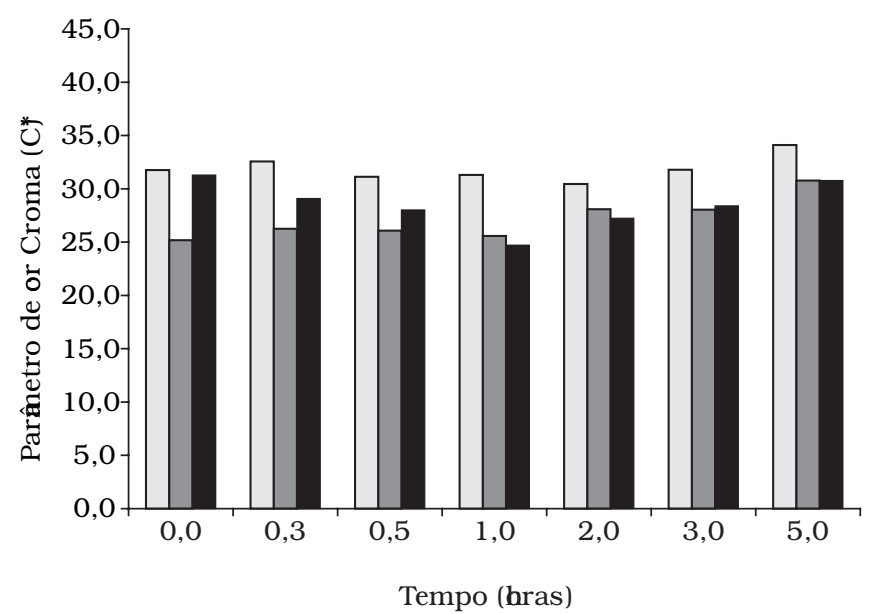

$$
\begin{aligned}
& \square 55^{\circ} \mathrm{ExP} \text { atm } \\
& \square 55^{\circ} \mathrm{BxP}=50 \mathrm{mbar} \\
& \square 55^{\circ} \mathrm{BxP}=100 \mathrm{mbar}
\end{aligned}
$$

Figura 5. Parâmetro de cor croma $\left(\mathrm{C}^{*}\right)$ variando-se a pressão de vácuo aplicada em soluções de $55^{\circ} \mathrm{Brix}$ a $20^{\circ} \mathrm{C}$.

\subsection{Tensão e deformação na ruptura}

As medidas da tensão na ruptura apresentaram um desvio elevado que pode ser atribuído à heterogeneidade entre as frutas e à falta de uniformidade em sua estrutura interna, uma vez que as propriedades reológicas de um material biológico são determinadas pela estrutura e pelos constituintes da parede celular, afetados pelas condições de processo e pelas pequenas variações no nível de maturação, variedade e época de colheita. Desvios elevados na determinação da tensão e deformação na ruptura também foram observados em trabalhos realizados com goiabas ${ }^{24}$, maçãs ${ }^{18}$ e melão ${ }^{11}$. Assim, o valor da tensão de ruptura foi apresentada normalizada, ou seja, os valores de tensão foram divididos pelos respectivos valores obtidos para a fruta fresca.

Analisando a Figura 6, verifica-se que, para os tratamentos em soluções de $45^{\circ} \mathrm{Brix}$ a $20^{\circ} \mathrm{C}$ em pressão atmosférica e com pulso de vácuo de 50 mbar, os valores obtidos de tensão de ruptura foram próximos aos da fruta fresca. Já para a aplicação de 100 mbar, observa-se um decréscimo da tensão de ruptura logo no início do processo osmótico, mais acentuado na temperatura de processo de $30{ }^{\circ} \mathrm{C}$. Essa diminuição na tensão pode ser atribuída à maior incorporação de sólidos causada pela aplicação de 100 mbar de vácuo e pelo aumento da temperatura, que provocaram o amolecimento dos tecidos, ocasionando a diminuição na tensão máxima de ruptura, em comparação à fruta fresca. Em relação à deformação (Figura 7), observou-se que todos os tratamentos foram afetados pelo processo, já que ocorreu o aumento da deformação na ruptura ao longo do tempo de processo sem, no entanto, apresentar tendências definidas entre si.

Nos processos realizados em soluções de $55^{\circ} \mathrm{Brix}$ a $20^{\circ} \mathrm{C}$, as amostras apresentaram valores de tensão de ruptura próximos da fruta fresca, indicando que essas condições contribuem para a manutenção das propriedades reológicas iniciais do

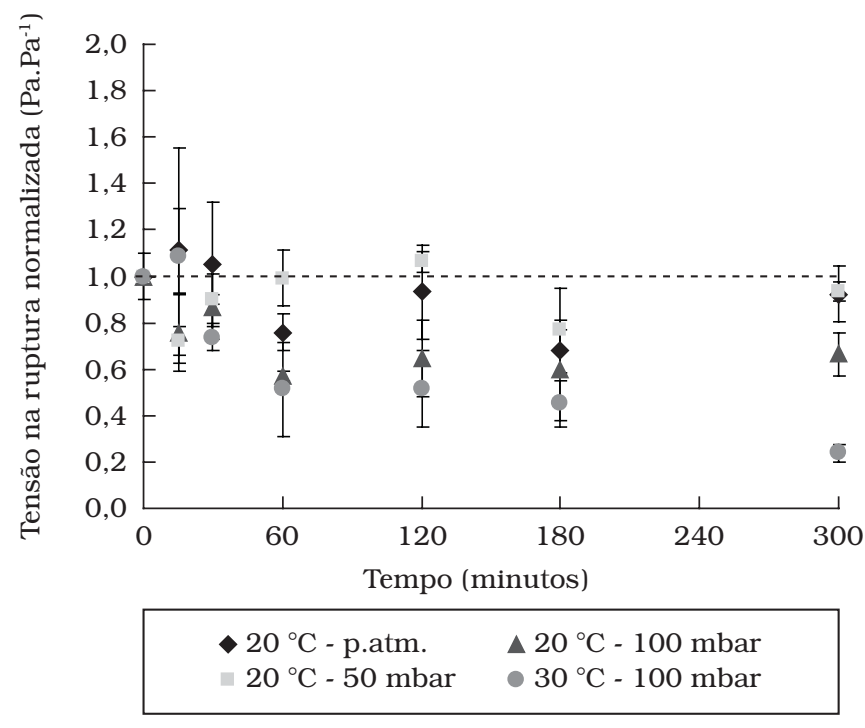

Figura 6. Tensão na ruptura normalizada $\left(\mathrm{Pa} \mathrm{Pa}^{-1}\right)$ variando-se a temperatura e a pressão para soluções a $45^{\circ} \mathrm{Brix}$.

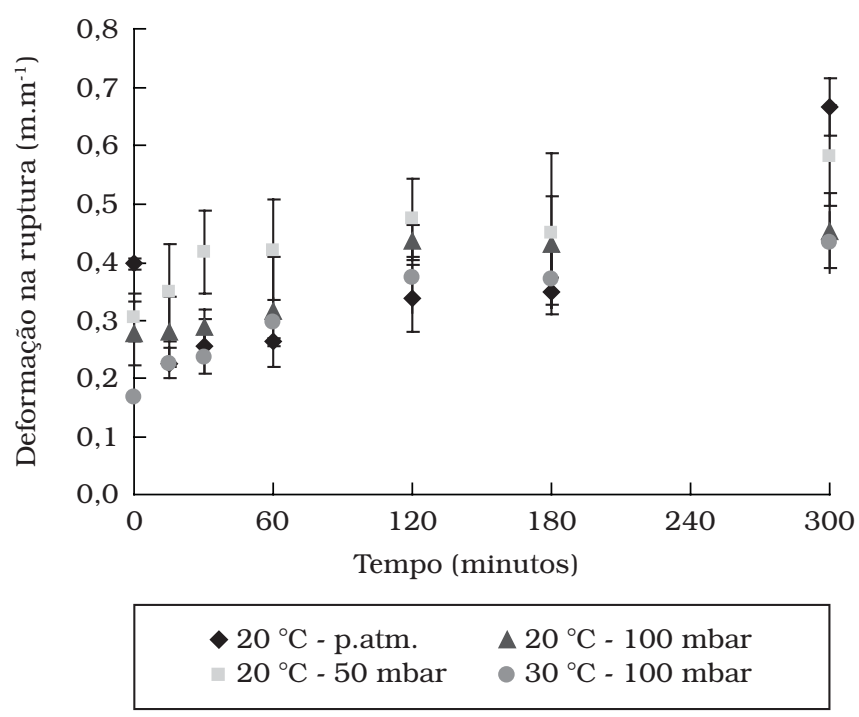

Figura 7. Deformacão na ruptura $\left(\mathrm{m} \cdot \mathrm{m}^{-1}\right)$ variando-se a temperatura e a pressão para soluções a $45^{\circ}$ Brix.

produto (Figura 8). O processo com aplicação de 50 mbar de pulso de vácuo apresentou valores inferiores de tensão na ruptura, porém, acima de 0,8 $\left(\mathrm{Pa}^{\mathrm{Pa}} \mathrm{Pa}^{-1}\right)$, e valores superiores de deformação na ruptura. Essa diferença pode ter sido causada pelas características da matéria-prima, que apresentou valores superiores de deformação na ruptura em comparação aos demais tratamentos (Figura 9).

O aumento da temperatura de 20 para $30{ }^{\circ} \mathrm{C}$ provocou um declínio na tensão na ruptura, originando um produto mais mole, com menor resistência à ruptura, como pode ser verificado pela Figura 8, que apresenta a cinética da tensão na ruptura normalizada para os processos a 20 e $30{ }^{\circ} \mathrm{C} \mathrm{em}$ soluções a $55^{\circ} \mathrm{Brix}$. Isto pode ter ocorrido pela degradação do tecido celular, causada pelo aumento da temperatura, o que 


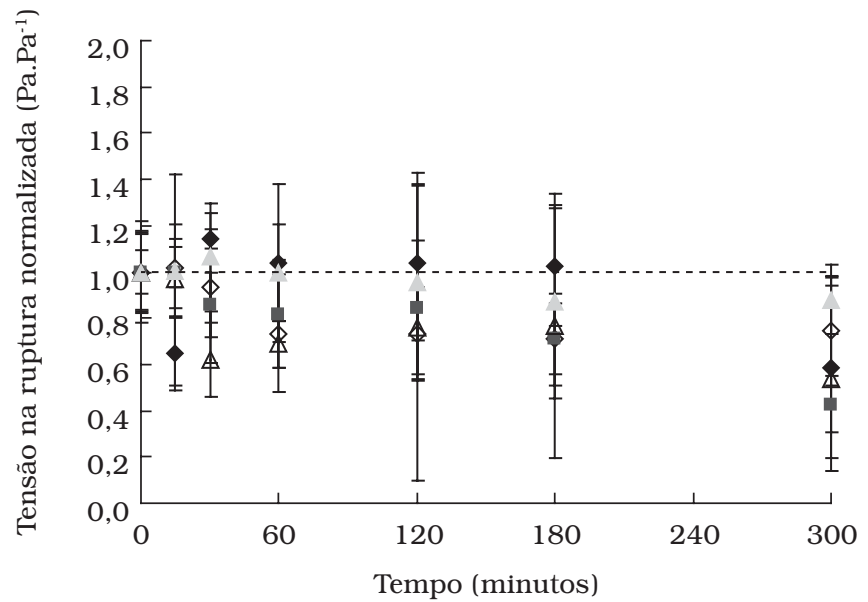

$20^{\circ} \mathrm{C}, 50$ mbar $\quad \triangle 30^{\circ} \mathrm{C}, 100 \mathrm{mbar}$ $20^{\circ} \mathrm{C}, 100 \mathrm{mbar}$

Figura 8. Tensão na ruptura normalizada $\left(\mathrm{Pa} \mathrm{Pa}^{-1}\right)$ variando-se a temperatura e a pressão para soluções a $55^{\circ} \mathrm{Brix}$.

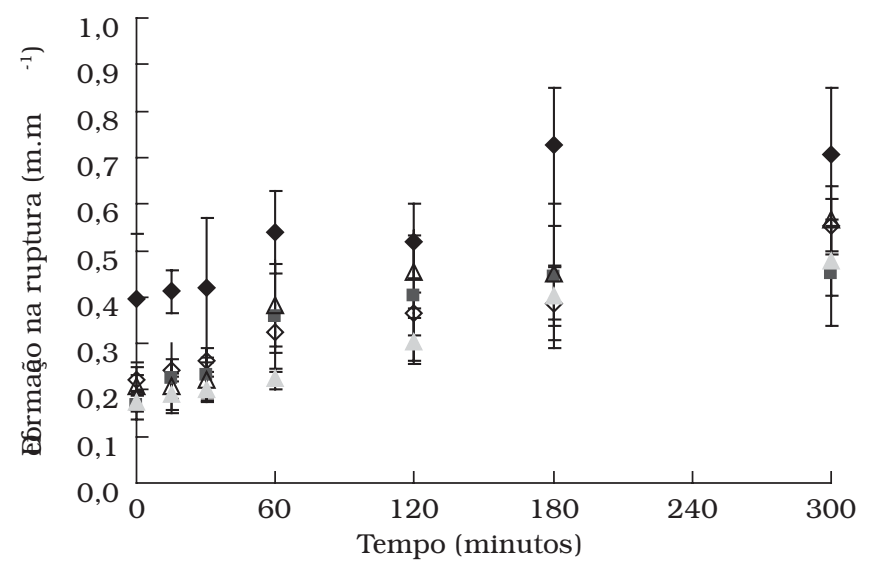
$20^{\circ} \mathrm{C}$, p.atm
$\diamond 30^{\circ} \mathrm{C}$, p.atm
- $20^{\circ} \mathrm{C}, 50$ mbar
$\triangle 30{ }^{\circ} \mathrm{C}, 100$ mbar
$20{ }^{\circ} \mathrm{C}, 100$ mbar

Figura 9. Deformação na ruptura $\left(\mathrm{m} \cdot \mathrm{m}^{-1}\right)$ variando-se a temperatura e a pressão para soluções a $55^{\circ}$ Brix.

permitiu uma maior penetração de solutos no produto ${ }^{17}$. No processo conduzido a $30^{\circ} \mathrm{C}$, a degradação do tecido celular, que permitiu uma maior penetração de solutos no produto, acarretou numa maior plasticidade da estrutura e, conseqüentemente, uma maior resistência à deformação, deixando o produto mais elástico em comparação aos processos a $20^{\circ} \mathrm{C}$.

Para o tratamento com solução de sacarose a $65{ }^{\circ} \mathrm{Brix}$, observa-se uma tendência ao aumento da tensão de ruptura para os processos a $20^{\circ} \mathrm{C}$ com as amostras apresentando uma característica mais acentuada de dureza, isto é, maior resistência à deformação e à ruptura (Figura 10). Soluções muito concentradas provocam uma baixa incorporação de sólidos e uma grande perda de água e, em conseqüência, apresentam um endurecimento da amostra, caracterizado pelo aumento da tensão máxima na ruptura, em comparação com as amostras in natura.

O tratamento a $30{ }^{\circ} \mathrm{C}$ foi mais eficiente na manutenção das propriedades reológicas já que o aumento da temperatura de 20 para $30{ }^{\circ} \mathrm{C}$ causa, além do amolecimento dos tecidos, um maior ganho de sólidos, que compensou o endurecimento causado pela grande perda de água da amostra em soluções de $65^{\circ}$ Brix.

Em relação à deformação (Figura 11), observou-se que todos os tratamentos foram afetados pelo processo, porém não apresentaram uma tendência em relação às variáveis de processo.

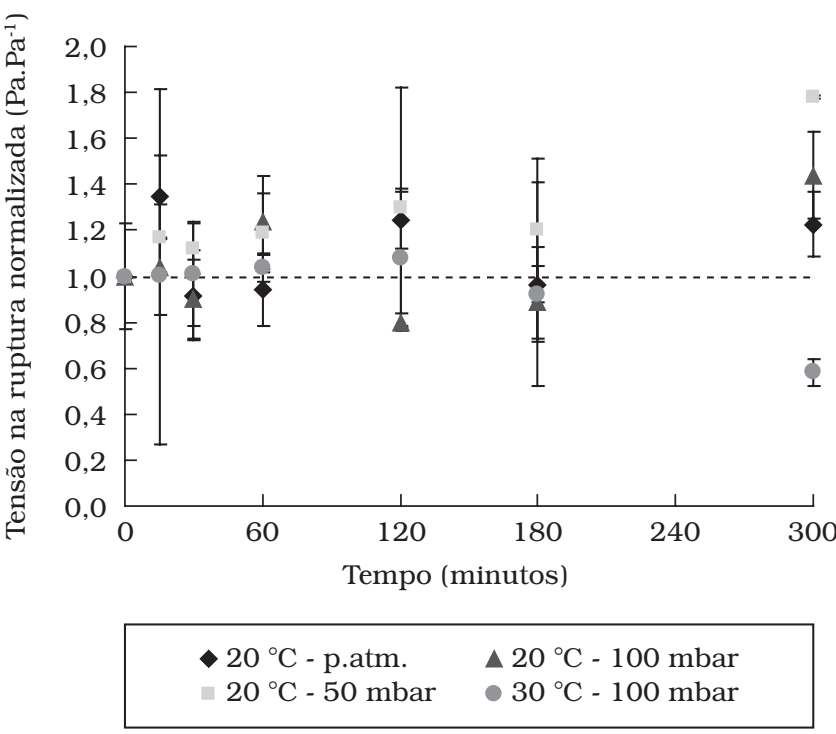

Figura 10. Tensão na ruptura normalizada $\left(\mathrm{Pa} \mathrm{Pa}^{-1}\right)$ variando-se a temperatura e a pressão para soluções a $65^{\circ} \mathrm{Brix}$

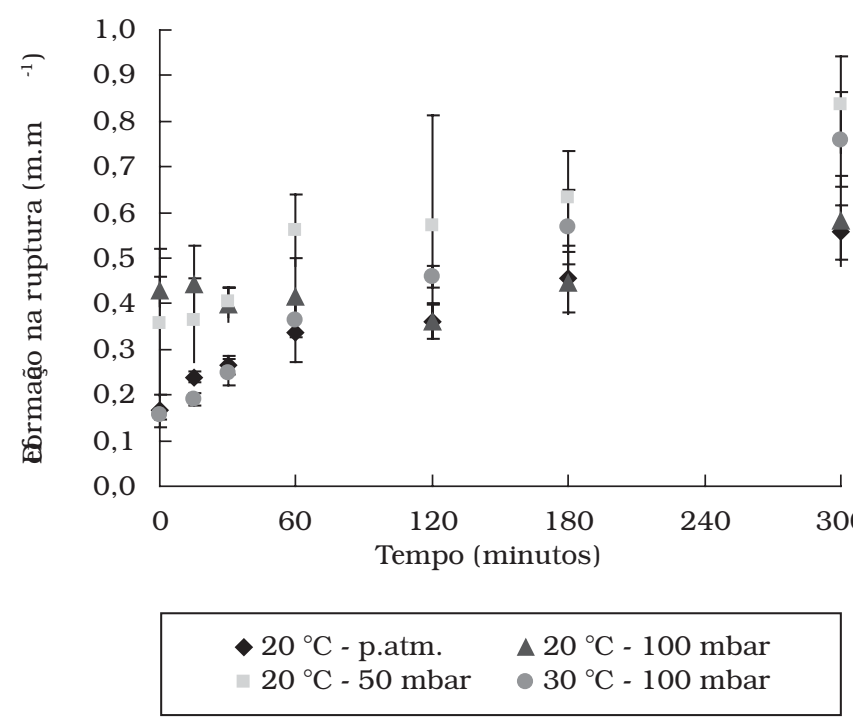

Figura 11. Deformação na ruptura $\left(\mathrm{m} . \mathrm{m}^{-1}\right)$ variando-se a temperatura e a pressão para soluções a $65^{\circ} \mathrm{Brix}$. 
O aumento da temperatura e o uso de concentrações mais baixas provocaram um declínio da tensão na ruptura. Nestas condições, houve uma maior incorporação de sólidos causando mudanças na estrutura e consequente amolecimento dos tecidos. MONSALVE-GONZÁLEZ et al. ${ }^{19}$ verificam que em maçãs desidratadas osmoticamente, a mudança de textura no processo osmótico é atribuída principalmente aos fluxos de perda de água e ganho de sólidos e a fatores químicos e enzimáticos, como a decomposição da pectina presente na parede celular.

As faixas de trabalho utilizadas para a aplicação do vácuo (variando de 0 a 100 mbar de vácuo por 10 minutos) podem não ter sido suficientes para provocar alterações na estrutura do material. Porém, observou-se que a aplicação do vácuo causou uma maior incorporação de solutos, principalmente nas concentrações mais baixas, e isso se refletiu nas propriedades reológicas do produto. Quando o tempo de vácuo é mais longo (mais que 15 minutos), possíveis danos mecânicos na disposição celular podem ocorrer, como a separação das células, associadas à deformação da amostra quando o pulso de vácuo é aplicado, provocando um amolecimento das amostras ${ }^{5,6}$. Na desidratação osmótica de kiwis a $30{ }^{\circ} \mathrm{C}$, CHIRALT et al. ${ }^{5}$ observaram que a aplicação do pulso de vácuo provocou uma maior redução da tensão na ruptura do que a observada nos processos conduzidos à pressão atmosférica. Os autores também relataram que longos tempos de imersão não são favoráveis à manutenção das propriedades reológicas do produto final.

De modo geral, é possível notar que o processo de desidratação osmótica nas condições realizadas não provocou grandes alterações na tensão de ruptura, quando comparada à fruta fresca. Este fato é favorável, pois se deseja que o processo de desidratação osmótica proporcione a manutenção das características de qualidade da fruta in natura, tais como textura e cor.

Considerando as situações estudadas, para a obtenção de fatias de manga osmoticamente desidratadas que combine uma maior perda de água, menor ganho de sólidos e manutenção das propriedades reológicas e de cor, a melhor condição de processo seria $65{ }^{\circ} \mathrm{Brix}, 30{ }^{\circ} \mathrm{C}$ e aplicação de 100 mbar de pulso de vácuo.

\section{Conclusões}

A desidratação osmótica foi afetada principalmente pela temperatura e concentração da solução osmótica. O aumento destes fatores proporcionou maiores taxas de perda de água e perda de peso e, conseqüentemente, favoreceu a depressão de $\mathrm{a}_{\mathrm{w}}$. Soluções mais viscosas, altas concentrações e baixas temperaturas foram eficientes em prevenir o ganho de sólidos.

A aplicação de pulso de vácuo na desidratação osmótica de fatias de manga proporcionou maior incorporação de solutos, com uma pequena contribuição na retirada de água. A aplicação de somente 50 mbar de vácuo não foi suficiente para causar alterações significativas nos processos estudados, em comparação ao processo à pressão atmosférica.
As propriedades reológicas estudadas foram afetadas durante o tratamento osmótico, sofrendo alterações por influência dos fluxos de transferência de massa ao longo da desidratação e das condições de processo. Entretanto, apesar de provocar mudanças na estrutura celular, o processo estudado apresentou-se como uma técnica eficiente para a obtenção de frutas a alta umidade, com manutenção dos parâmetros de qualidade avaliados (tensão e deformação na ruptura, croma e luminosidade).

\section{Agradecimentos}

Os autores agradecem ao CNPq e ao PIBIC/CNPq pela concessão das bolsas de doutorado e iniciação científica e à FAPESP e Capes-Grices pelo apoio financeiro.

\section{Referências bibliográficas}

1. ALZAMORA, S. M.; GERSCHENSON, L. N.; VIDALES, S. L.; NIETO, A. Structural Changes in the Minimal Processing of Fruits: Some Effects of Blanching and Sugar Impregnation. In: FITO, P; ORTEGA-RODRIGUES, E.; BARBOSA-CANOVAS, G. Food Engineering, 2002. New York: Chapman and Hall, 1997. p. 117-139.

2. AOAC. (Association of Official Analytical Chemists). Official methods of analysis. $16^{\circ}$ edição, Arlington, 1995.

3. BARAT, J. M.; CHIRALT, A.; FITO, P. Effect of osmotic solution concentration, temperature and vacuum impregnation pretreatment on osmotic dehydration kinetics of apple slices. Food Science and Technology International, v. 7 , n. 5, p. 451-456, 2001.

4. CALVO, C. DURÁN, L. Propriedades Físicas II - Ópticas y Color. In: AGUILERA, J. M. Temas en Tecnología de Alimentos, México: Instituto Politécnico Nacional, 1997. v. I, cap. 7, p. 261-288.

5. CHIRALT, A.; MARTINÉZ,-NAVARRETE, N.; MARTÍNEZMONZÓ, J.; TALENS, P.; MORAGA, G.; AYALA, A.; FITO, P. Changes in mechanical properties throughout osmotic process Cryoprotectant effect. Journal of Food Engineering, v. 49, n. 1, p. 129-135, 2001.

6. CHIRALT, A., TALENS, P. Physical and chemical changes induced by osmotic dehydration in plant tissues. Journal of Food Engineering, v. 67, n. 1, p. 167-177, 2005

7. COSTELL, E.; FISMAN, S.M.; DURÁN, L. Propiedades Físicas I - Reologia de Sólidos y Textura. In: AGUILERA, J.M. Temas em Tecnología de Alimentos, México: Instituto Politécnico Nacional, 1997. v. I, cap. 6, p. 215-260.

8. ESCRICHE, I.; GARCIA-PINCHI, R.; ANDRÉS, A.; FITO, P. Osmotic dehydration of kiwifruit (Actinidia chinensis): fluxes and mass transfer kinetics. Journal of Food Process Engineering, v. 23, n. 3, p. 191-205, 2000.

9. ESCRICHE, I.; GARCIA-PINCHI, R.; CAROT, J. M.; SERRA, J. A. Comparison of must and sucrose solutions to obtain high quality minimally processed kiwi fruit (Actinidia chinensis P.) slices. International Journal of Food Science and Technology, v. 37, n.1, p. 87-95, 2002.

10. FAO. Agricultural production (2003). Disponível em: <http:// faostat.fao.org/faostat/collections?subset=agriculture/ $>$. Acesso em: 10 de out. 2006.

11. FERRARI, C. C. Estudo da transferência de massa e qualidade do melão desidratado osmoticamente em soluções de sacarose e maltose. Campinas, 2005. 98 p. Dissertação (Mestre 
em Engenharia de Alimentos) - Faculdade de Engenharia de Alimentos, Universidade Estadual de Campinas.

12. FERRARI, C. C; RODRIGUES, L. K.; TONON, R. V.; HUBINGER, M. D. Cinética de transferência de massa de melão desidratado osmoticamente em soluções de sacarose e maltose.Ciência e Tecnologia de Alimentos, v. 25, n. 3, p. 564-570, 2005.

13. FITO, P.; Modeling of vacuum osmotic dehydration of food. Journal of Food Engineering, v. 22, n. 1-4, p. 313-328, 1994.

14. FITO, P.; ANDRÉS, A.; CHIRALT, A.; PARDO, P. Coupling of Hydrodinamic and Deformation-Relaxation Phenomena during vacuum treatments in solid porous food-liquid systems. Journal of Food Engineering, v. 27, n. 3, p. 229-240, 1996.

15. GIRALDO, G.; TALENS, P.; FITO, P.; CHIRALT, A. Influence of sucrose solution concentration on kinetics and yield during osmotic dehydration of mango. Journal of Food Engineering, v. 58, n. 1, p. 33-43, 2003.

16. LAZARIDES, H. N. Reasons and possibilities to control solids uptake during osmotic treatment of fruits and vegetables. In: FITO, P.; CHIRALT, A.; BARAT, J. M.; SPIESS, W. E. L.; BEHSNILIAN, D. (Eds.). Osmotic dehydration \& Vacuum impregnation. Lancaster, Pennsylvania: Technomic Publishing Company, 2001. p. 33-42.

17. LAZARIDES, H. N.; KATSANIDIS, E.; NICKOLAIDIS, A. Mass transfer kinetics during osmotic preconcentration aiming at minimal solid uptake. Journal of Food Engeneering, v. 25, n. 2, p. 151-166, 1995.

18. LEWICKI, P. P.; LUKASZUK, A. Effect of Osmotic Dewatering on Rheological Properties of Apple Subjected to Convective Drying. Journal of Food Engineering, v. 45, n. 3, p. 119-126, 2000.

19. MONSALVE-GONZÁlEZ, A. M.; BARBOSA-CÁNOVAS, G. V.; CAVALIERI, R. P. Mass Transfer and Textural Changes during Processing of Apples by Combined Methods. Journal of Food Svience, v. 58, n. 5, p. 1118-1124, 1993.

20. MÚJICA-PAZ, H.; VALDEZ-FRAGOSO, A.; LÓPEZ-MALO, A.; PALOU, E.; WELTI-CHANES, J. Impregnation properties of some fruits at vacuum pressure. Journal of Food Engineering. v. 56, n. 4, p. 307-314, 2003.

21. PANADÉS, G.; CHIRALT, A.; FITO, P.; RODRÍGUEZ, I.; NUÑES, M.; ALBORS, A.; JIMÉNEZ, R. Influence of operating conditions on sensory quality of minimally processed osmotically dehydrated guava. Journal of Food Quality, v. 26, n. 2, p. 91-103, 2003.

22. PANADÉS, G.; FITO, P.; AGUIAR, Y.; VILLAVICENCIO, M. N.; ACOSTA, V. Osmotic dehydration of guava: Influence of operating parameters on process kinetics. Journal of Food Engineering, v. 72, n. 4, p. 383-389, 2006.

23. PEREIRA, L. M. Acondicionamento de Goiabas Minimamente Processadas por Desidratação Osmótica em Embalagens sob Atmosfera Modificada Passiva. Campinas, 2002, 159 p. Dissertação (Mestre em Engenharia de Alimentos) - Faculdade de Engenharia de Alimentos, Universidade Estadual de Campinas (UNICAMP).

24. PEREIRA, L. M.; RODRIGUES, A. C. C.; SARANTÓPOULOS, C. I. G. L.; JUNQUEIRA, V. C. A.; CUNHA, R. L.; HUBINGER, M. D. Influence of Modified Atmosphere Packaging and Osmotic Dehydration of Minimally Processed Guavas. Journal of Food Science, v. 69, n. 4, p. 172-177, 2004.

25. RODRIGUes, A. C. C.; CUNHA, R. L.; HUBINGER, M. D. Rheological properties and colour evaluation of papaya during osmotic dehydration processing. Journal of Food Engineering, v. 59, n. 2-3, p. 129-135, 2003.

26. SANJINEZ-ARGANDOÑA, E. J.; HUBINGER, M. D.; MENEGALLI, F. C. Effect of osmotic dehydration on colour and mechanical properties of dried guavas. In: International Drying Symposium, 13, 2002, Beijing, China. Proceedings..., Beijing, 2002, v. B, p. 968-976 (CD-rom).

27. SERENO, A. M.; HUBINGER, M. D.; COMESAÑA, J. F.; CORREA, A. Prediction of water activity of osmotic solutions. Journal of Food Engineering, v. 49, n. 2-3, p. 103-114, 2001a.

28. TACO - Tabela Brasileira de Composição de Alimentos/NEPA - UNICAMP 2a . Edição. Campinas: NEPA-UNICAMP, 2006. Disponível em: http://www.unicamp.br/nepa/taco/. Acesso em 15 jan. 2006.

29. TALENS, P.; MARTÍNEZ-NAVARRETE, N.; FITO, P.; CHIRALT, A. Changes in Optical and Mechanical Properties during Osmodehydrofreezing of Kiwi Fruit. Innovative Food Science \& Emerging Technologies, v. 3, p. 191-199, 2002.

30. TORRES, J. D.; TALENS, P.; ESCRICHE, I.; CHIRALT, A. influence of process conditions on mechanical properties of osmotically dehydrated mango. Journal of Food Engineering, v. 74, n. 2, p. 240-246, 2006.

31. UDDIN, M. B.; AINSWORTH, P.; IBANOGLU, S. Evaluation of mass exchange during osmotic dehydration of carrots using response surface methodology. Journal of Food Engineering, v. 65, n. 4, p. 473-477, 2004.

32. VIVANCO PEZANTES, D. Estudo das operações combinadas da desidratação osmótica a vácuo, defumação líquida e secagem em filés de bonito (Sarda sarda). Campinas, 2006. 223 p. Tese (Doutor em Engenharia de Alimentos) - Faculdade de Engenharia de Alimentos, Universidade Estadual de Campinas (UNICAMP).

33. WOLF, W.; SPIESS, W. E. L.; JUNG, G. Sorption isotherms and water activity of food material. New York, Elsevier Science, 1985, $239 \mathrm{p}$. 